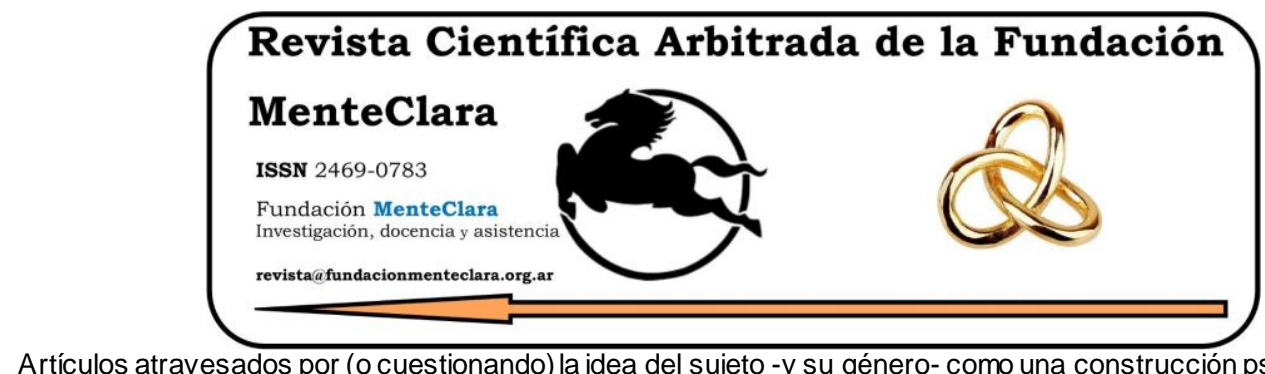

Artículos atravesados por (o cuestionando) la idea del sujeto -y su género- como una construcción psicobiológica de la cultura. Articles driven by (or questioning) the idea of the subject -and their gender- as a cultural psychobiological construction.

Vol. 7 (2022), enero-diciembre ISSN 2469-0783

https: / / datahub.io/ dataset/2022-7-e267

\title{
ANÁLISIS DE LA INTELIGENCIA EMOCIONAL DE LOS ESTUDIANTES UNIVERSITARIOS EN EL AULA DE CLASES
}

\author{
ANALYSIS OF THE EMOTIONAL INTELLIGENCE OF UNIVERSITY STUDENTS IN \\ THE CLASSROOM
}

Yan An Cornejo M ontoya yancornejo@yahoo.com Ingeniera de Sistemas, Magíster en Seguridad Informática Aplicada, Consultora acreditada OIT, Diplomatura en Pedagogia Universitaria, Investigadora independiente, Ecuador

Dennis Morán dr.dennismoran@gmail.com

Doctor en Medicina General, Diplomatura Superior en enfermedades inmunodeficientes VIH/SIDA, Docente de la Facultad de Enfermería de la Universidad UNEMI, Ecuador

Sofia Alejandra Garcia Cornejo sagc2298@outlook.com Lcda. en Gestión Empresarial Internacional, Investigadora independiente, Ecuador

Cómo citar este artículo / Citation: Cornejo, Y; Morán, D; García, S. (2022). Análisis de la inteligencia emocional de los estudiantes universitarios en el aula de clases. Revista Científica Arbitrada de la Fundación MenteClara, Vol. 7 (267). DOI:

https://doi.org/10.32351/rca.v7.267

Copyright: (C) 2022 RCAFMC. Este artículo de acceso abierto es distribuido bajo los términos de la licencia Creative Commons Attribution 4.0 International License (CC BY 4.0). Recibido: 18/01/2022. Aceptado: 03/02/2022 Publicación online: 11/02/2022

Conflicto de intereses: Ninguno que declarar.

\section{Resumen}

Abordar este tema motiva a una revisión sistemática de la neurodidáctica relacionada en el proceso de aprendizaje, la misma que tienen relación directa no solo con la memoria sino con estimulos emocionales. El aprendizaje inicia en el hipocampo y en la amígdala colaborando en la evaluación de los estímulos emocionales. Mediante una investigación empírica con una muestra integrada por 160 participantes universitarios de la UNEMI, se les aplicó el instrumento ECI, cuyos resultados permitieron analizar cómo los estudiantes manejan sus habilidades de inteligencia 
emocional en el aula. Las estrategias didácticas que se utilizan en el aula tien en la capacidad de favorecer el aprendizaje de los estudiantes o de impedirlo, en función de la gestión de las emociones que el maestro realice. El artículo se elaboró sobre la base de la revisión de literatura relacionada con el funcionamiento del cerebro emocional, y la forma en que las emociones influyen en el aprendizaje humano.

\begin{abstract}
Addressing this issue motivates a systematic review of neurodidactics related to the learning process, which are directly related not only to memory but also to emotional stimuli. Learning begins in the hippocampus and amygdala collaborating in the evaluation of emotional stimuli. Through empirical research with a sample made up of 160 university participants from UNEMI, the ECI instrument was applied, the results of which made it possible to analyze how students manage their emotional intelligence skills in the classroom. The didactic strategies that are used in the classroom have the capacity to favor the learning of the students or to prevent it, depending on the management of the emotions that the teacher carries out. The article was developed based on a review of the literature related to the functioning of the emotional brain, and the way in which emotions influence human learning.
\end{abstract}

Palabras Claves: Inteligencia Emocional; aprendizaje; neuroeducación

Keyw ords: Emotional Intelligence; learning; neuroeducation 


\section{Introducción}

El interés sobre el estudio de la Inteligencia emocional en aulas universitarias surge como interrogante por las interacciones del conglomerado de docentes y alumnos que denotan a simple vista una serie de vacíos educativos que develan el quehacer del área educativa, cuyo propósito se ve inmerso en el desempeño del estudiantado, debido a la calidad de conocimientos que demuestran en el desarrollo de clases tal como lo menciona (Godoy \& Sánchez, 2020).

La Inteligencia Emocional (IE) como constructo tiene su origen a partir de los trabajos de Salovey y Mayer en 1990, quienes aseguraban que la IE es la habilidad para reconocer a las emociones, así como de razonar y solucionar los problemas de acuerdo con las emociones presentadas (Estrada, Moysén, Balcazar, \& Garay, 2016).

Montenegro (2020) afirma que el concepto de Inteligencia emocional propuesto por Salovey \& Mayer en 1990 surgieron de las temáticas de inteligencias intrapersonal e interpersonal concebidas en la teoría de las inteligencias múltiples de que menciona Gardner citado por Trujillo y Rivas en 2005, refiriéndose a la capacidad de evaluar, regular y comprender las propias emociones y de las de otros seres humanos con el objetivo de promover el desarrollo emocional e intelectual descrito por Mayer \& Salovey (1997).

Así también Montenegro (2020) que cita a Vasefi, Dehghani, \& Mirzaaghapoor (2018) reitera que la inteligencia emocional conduce a un efecto positivo del éxito personal, y al mejoramiento de las relaciones personales y laborales, necesarias para el éxito profesional, situaciones que oportunamente permiten el ajuste en la universidad y que facilita el logro académico. 
La Inteligencia emocional se refiere al manejo de las emociones, empatía, así como conocerse a sí mismo, el autocontrol y como comprender las actuaciones de quienes nos rodean, que conlleve a buenas actitudes, que generen comportamientos de cordialidad que eviten situaciones de hostilidad y agresividad (Cárdenas \& Rodríguez, 2020).

Muchas investigaciones sobre la temática de Inteligencia Emocional afianzan un mejor desempeño tanto en el ámbito profesional, condiciones laborales, así como afrontar con mejores estrategias, manejando mejores factores de estrés enfrentando nuevos desafios con mejor eficacia (Castillo, García, Pena, \& Galán, 2017).

El presente estudio se orienta a identificar la importancia que tienen las emociones en los jóvenes y que deben ser atendidas y desarrolladas para obtener un buen desempeño académico del manejo de la inteligencia emocional en los estudiantes universitarios de la Facultad de Enfermería de la Universidad Estatal de Milagro (UNEMI), ubicada en la ciudad de Milagro Provincia del Guayas, con la finalidad de analizar la convivencia de estudiantes y docentes, donde se produce la socialización emocional (Cáceres, García, \& García, 2020).

\subsection{Bases Fundamentales de la Inteligencia emocional}

Los principales modelos teóricos que se desprenden del estudio de la inteligencia emocional son: el modelo de habilidades de Mayer \& Salovey; el modelo mixto para la interacción inter e intrapersonal de Goleman y el modelo mixto de las habilidades y la personalidad de Bar-on (Bueno, 2019; Trujillo, \& Rivas, 2005; Macavilca, 2020). 
Figura 1. Modelos de Inteligencia Emocional

\begin{tabular}{|c|c|c|c|}
\hline Modelos & $\begin{array}{c}\text { Mayer-Salovey } \\
\text { (199) }\end{array}$ & $\begin{array}{c}\text { Goleman } \\
\text { (19:5) }\end{array}$ & $\begin{array}{c}\operatorname{8an} 0 \mathrm{n} \\
(200)\end{array}$ \\
\hline ¿Qué abarca? & $\begin{array}{l}\text { Se maneja la emoción de } \\
\text { manera individual por lo } \\
\text { que evalía y atende el } \\
\text { estado de animo, lo } \\
\text { reftexiona y se expresa } \\
\text { con precisión }\end{array}$ & $\begin{array}{l}\text { Ueliza el reconocimiento y } \\
\text { reguladión de las emodiones, } \\
\text { asi como también el trabajo } \\
\text { en equipo ya que maneja la } \\
\text { empatia al reconocer las } \\
\text { emociones de los demás. }\end{array}$ & \begin{tabular}{|l|} 
a influir de manera \\
individual con los \\
pensamiertos para \\
camblar su emodion y/o \\
estado de animo. \\
Atender las demandas del \\
medio ambiente, \\
capacidad de adaptarse y \\
manefar el estrés
\end{tabular} \\
\hline Componentes & $\begin{array}{l}\text { 1) Percibir, apreciar y } \\
\text { expresar las } \\
\text { emociones. } \\
\text { 2) Asimlar las emociones. } \\
\text { 3) Entender y analizar las } \\
\text { emociones. } \\
\text { 4) Reflexionar y regular } \\
\text { las emociones }\end{array}$ & $\begin{array}{l}\text { 1) Auto-conclencia (Self- } \\
\text { awareness) } \\
\text { 2) Auto-dirección (Self- } \\
\text { drecition) } \\
\text { 3) Aottudes Sociales (Social- } \\
\text { awareness) } \\
\text { 4) Relaciones de Direccion } \\
\text { (Relationship managment) }\end{array}$ & $\begin{array}{l}\text { 1) Intrapersonal } \\
\text { 2) Interpersonal } \\
\text { 3) Manejo de estrés } \\
\text { 4) Estado de animo } \\
\text { 5) Adaptabilidad }\end{array}$ \\
\hline Critica & $\begin{array}{l}\text { Es un método de } \\
\text { conocimiento emocional } \\
\text { individual muy completo, } \\
\text { sin embargo es rilgido y } \\
\text { con mucho componente } \\
\text { meta cognitivo. }\end{array}$ & $\begin{array}{l}\text { Es un metododo ưfilizado mas } \\
\text { comunnmente para empresas, } \\
\text { a nivel educativo es } \\
\text { superficial. }\end{array}$ & $\begin{array}{l}\text { E modelo Ideal para } \\
\text { usarse dentro del salón de } \\
\text { clases, sin embargo es } \\
\text { muy complejo. }\end{array}$ \\
\hline
\end{tabular}

Fuente:http://elpoderdelainteligencia.blogspot.com/2014/09/modelos-de-inteligenciaemocional.html Autor: Eileen Silva Navarro

Como se aprecia en la figura 1 se muestra la tabla comparativa de los tres modelos de inteligencia emocional más reconocidos, aunque con el pasar del tiempo han surgido nuevos autores e investigadores de la IE.

La Inteligencia Emocional vista desde el modelo de Mayer \& Salovey, la más utilizada como sustento teórico (Alejos \& Cambero, 2017), radica en que las emociones inician como una propuesta tentativa, donde las personas utilizan las emociones para resolver problemas de cualquier ámbito, así también menciona su relación con elliderazgo organizacional, relaciones interpersonales y el campo educativo y esto ocasionó que surgieran algunos detractores como el escritor Locke (2005) que aseguraba que el concepto de inteligencia emocional no era válido por tener amplias definiciones que daban lugar a confusiones (Bueno, 2019).

El concepto de Inteligencia emocional desde el modelo de Goleman (1996) se produce por las múltiples investigaciones que se llevaron a cabo 
durante una década, en donde (Bueno, 2019) menciona aspectos a considerar como:

a) El hecho de conocerse a sí mismo que consiste en entender lo que está sintiendo y porqué experimenta dicha emoción.

b) Manejo de sus emociones, es decir ejercer dominio sobre sí mismo y aprender a manejarlas en diferentes situaciones con el fin de mantener relaciones interpersonales sanas.

c) Automotivación para alcanzar los objetivos en nuestra vida como el logro de metas laborales o de estudio.

d) Reconocimiento de las emociones de nuestro entorno cuya base es la empatía con altruismo reconociendo emociones imperceptibles en los demás con el finde mejorar la convivencia.

e) Establecer relaciones con el fin de manejar las relaciones sociales que afianzan el liderazgo, la popularidad social y la eficiencia intrapersonal.

El último modelo es el de Bar-On en 1997 también conocido como inteligencia emocional y social relacionadas a los conocimientos y habilidades que sirven para afrontar de manera efectiva las situaciones del medio (Alejos \& Cambero, 2017; Fernández \& Inga, 2019), así también las habilidades que menciona Bar-On (1997) se relacionan con las que propone Goleman (1996) y con los autores Mayer \& Salovey como son: la autoconciencia, la comprensión, el control y expresión de las emociones (Cárdenas \& Rodríguez, 2020).

En ese sentido Cáceres (2020) cita a Fragoso-Luzuriaga (2015), ratificando su criterio en cuanto al modelo de Bar-On (1997) que está conformado por cinco (5) componentes:

- El componente intrapersonal que genera autoconocimiento 
- El componente interpersonal es la capacidad para manejar emociones fuertes producidas en otros y por otros

- El componente del manejo del estrés es la manera positiva de ver un problema que genera presión

- El componente de estado de ánimo

- El componente de adaptabilidady ajuste se refieren esta dos últimas a la manera de regular los estados anímicos pese a las diferentes adversidades.

Así también, Cazallo et al. (2020) cita a Fragoso-Luzuriaga (2015), afirmando que el modelo de Goleman, así como el de Mayer \& Salovey en 1997, se han perfeccionado en el tiempo, donde coinciden con sus perspectivas de la IE tal como el autor Bar-On (1997), que menciona a dos grupos, dónde el primero contiene las habilidades básicas como: la autoevaluación, la autoconciencia emocional, asertividad, la empatía, las relaciones sociales, el afrontamiento de presiones, el control de impulsos, el examen de realidad, la flexibilidad y la solución de problemas y el segundo grupo se refiera a las capacidades facilitadoras como: optimismo, la autorrealización, la alegría, la independencia emocional y la responsabilidad social, donde afirma que sin las capacidades básicas no existirian las capacidades facilitadoras.

Howard Gardner al desarrollar la teoría de inteligencias múltiples en 1983 (Cárdenas \& Rodríguez, 2020), creó el término inteligencia emocional la cual la define como aquella capacidad de motivarse a sí mismo, tener constancia a pesar de las posibles frustraciones, controlar los impulsos, manejo de estados de ánimo, con trolar estados de angustia, así como ser empáticos y confiar en los demás (García A, 2020). 
García A (2020) afirma también que para comprender este concepto, Coleman en 1996 mencionó que tanto las emociones con la inteligencia tienen un origen cerebral, en donde la amígdala es una estructura subcortical ubicada en el cerebro y que en la actualidad se la considera una estructura límbica relacionada a procesos de aprendizaje y memoria, y que está implícita en respuestas emocionales: los sentimientos, la expresión de la emoción, los recuerdos de las emociones y el reconocimiento de los signos de la emoción de los demás" (Benavidez \& Flores, 2019) que cita a Aguilar (2011).

A su vez Benavidez \& Flores (2019) reiteran en su investigación que hay una teoría del cerebro propuesta por el autor MacLean, ver figura 2, y retomada por Beauport que afirman que el ser humano tienen un sinnúmero de capacidades que están interconectadas y se complementan, la cual permite que se analice el comportamiento humano de manera integral donde la cognición, la emoción y la conducta humana trabajan al unísono e influyen definitivamente en el desempeño del sujeto en cualquier ámbito; en donde el cerebro emocional (también llamado cerebro mamífero) contiene al cerebro límbico que está ubicado detrás de la nariz hasta los occipitales, relacionados por ende a la respiración, rabia, amor, estados de ánimo y el afecto.

Figura 2 Cerebro Triuno de Paul McLean

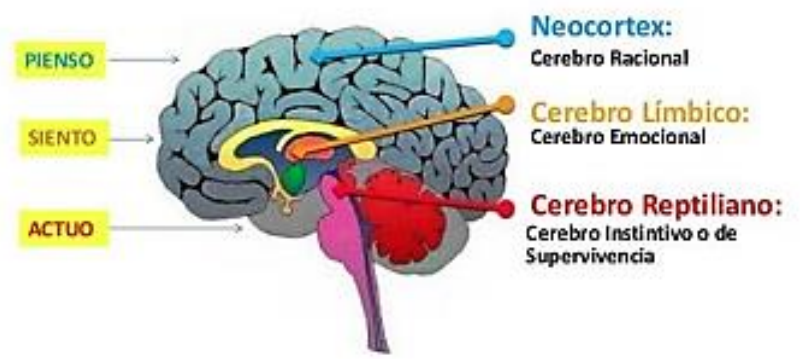

Fuente Benavidez \& Flores (2019) 
Benavidez \& Flores (2019) también afirman que el cerebro emocional está conformado por el hipocampo, la amígdala cerebral, el hipotálamo, el fórnix y los cuerpos mamilares, por lo que es muy importante el sistema limbico, debido a que es la responsable de asociar las cargas emocionales a los recuerdos a largo plazo, evitar experiencias dolorosas o por el contrario repetir situaciones que hayan provocado placer o alegria, tal es así que aquellos individuos que presentan alteraciones en la amígdala pierden la respuesta al temor y son agresivas (Gutiérrez S, Zamora L, Fouilloux, \& Petra, 2021).

El sistema límbico está conformado por algunos componentes funcionales como se lo aprecia en la figura 3 (Saavedra et al., 2015).

Figura 3. Componentes estructurales y funcionales del sistema límbico

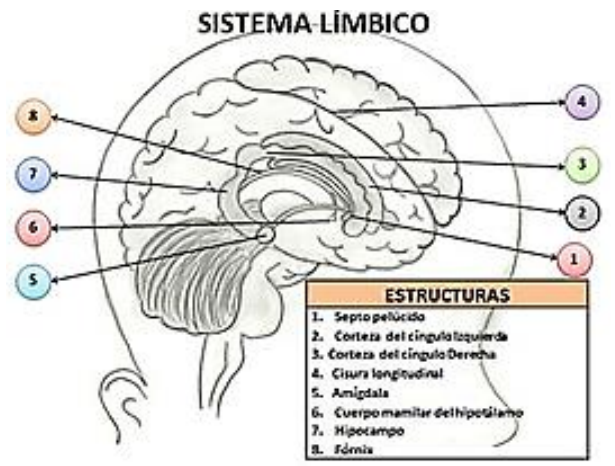

Fuente Saavedra, Díaz C, Zúñiga, Navia, \& Zamora (2015)

La palabra límbico tiene como significado borde o margen, y se lo vincula con los estados de la emoción visceral, cognición y comportamiento y que, por estudios realizados, el sistema límbico se lo asocia a estados como: la adicción, el apego, la recompensa, el placer; si se extirpa la amígdala se dificultaría el aprendizaje mediante recompensa y castigo (Saavedra et al., 2015). 
Las emociones experimentadas por el ser humano son muchas, tal como las define Montenegro (2020), donde se encuentran las emociones primarias: miedo, Ira, alegria, tristeza, disgusto y sorpresa, que van en conjunto con gestos faciales y respuestas vocales, motoras, endocrinas y autonómicas; así también están las emociones secundarias: calma, vergüenza, envidia, culpa, depresión etc., y que son asociadas a relaciones interpersonales (Saavedra et al., 2015).

\section{La neurociencia, neuroeducación y aprendizaje}

Las neurociencias son un conjunto de ciencias que estudian el sistema nervioso, así como la relación entre la conducta y el aprendizaje, siendo la neurociencia la disciplina encargada de estudiar al cerebro y que éste da origen a la conducta y al aprendizaje, así también estudia la plasticidad del sistema nervioso, el ambiente del aula, la motivación, la atención, las emociones y a la memoria, factores fundamentales en el proceso de enseñanza - aprendizaje (Benavidez \& Flores, 2019).

Mientras que la neuroeducación es la relación entre enseñanza, aprendizaje tema muy implicado a la escolarización, cuyo objetivo es fomentar la memoria de los estudiantes por medio de estrategias didácticas con apoyo a la docencia, donde se fortalezcan los aspectos positivos y se minimicen los negativos (Saavedra et al., 2015).

Y es así como la neurociencia siendo una disciplina que estudia la plasticidad del sistema nervioso se lo relaciona a la educación, ya que comprende la capacidad que tiene el cerebro de responder a los cambios que le rodean y que por ende mientras más aprende adquiere más conocimientos, por lo que la plasticidad del sistema nervioso denota más posibilidades de aprender cuando se encuentra en un ambiente de clases más relajado, pero enfrentando desafios y que por el contrario en 
situaciones de amenazas, desinterés, falta de atención ocasionan inhibición del aprendizaje y como cada cerebro es diferente según el estímulo del mismo será enriquecido o empobrecido, y los gustos o aversiones responderán a las necesidades de cada persona (Benavidez \& Flores, 2019; Araya P, \& Espinoza, 2020).

De este modo los aportes de la neurociencia han demostrado que la inteligencia emocional es un conjunto de habilidades distintas a las habilidades cognitivas, y que es importante reconocer a las emociones tales como: la tristeza, miedo y alegría como un lenguaje que relaciona la mente-cerebro-cuerpo (Leggiero, s.f); es decir que la emoción surge cuando la mente y el cuerpo se juntan lo que significa que la mente se refleja en el cuerpo (Viciana, Cano, Chacón, Padial, \& Martínez, 2017).

\section{Método}

\subsection{Procedimiento de búsqueda}

Para lograr una estructura coherente de esta investigación, se estableció esta revisión sistemática que recoge los estándares sobre la realización en ciencias de la salud de Perestelo-Pérez (2013). La búsqueda bibliográfica se ha realizado en las siguientes bases de datos: Researchgate, Latindex, Scielo y Dialnet. Esta búsqueda se llevó a cabo durante los meses julio y agosto de 2021, prestando una mayor atención a aquellos artículos cuyo impacto científico ha sido mayor. Así mismo, la búsqueda se limitó a los últimos cinco años, desde 2016 hasta 2021. Las palabras clave que se usaron para la búsqueda fueron "Emotional Intelligence", “academic performance" y "university students"; lo que sería igualmente "Inteligencia Emocional", "rendimiento académico" y "estudiantes universitarios" en el caso de los artículos titulados en castellano (Rodríguez, Rodríguez, Rodríguez G, \& Bohórquez, 2021). 


\subsection{Criterios de inclusión y exclusión}

Para la selección de los trabajos se tuvo en cuenta: (a) que los participantes fueran estudiantes universitarios independientemente de su edad y sexo; (b) que los estudios fueran empíricos; (c) que contaran con instrumentos validados estadísticamente; (d) que estuvieran publicados en los últimos diez años; y (e) que solamente se centraran en medir inteligencia emocional sin añadir otra clase de variables Como criterios de exclusión no se consideraron los estudios que no incluian datos relevantes como el número de participantes o fundamentos sobre la fiabilidad de los instrumentos, estudios cuyo carácter fuera de tipo cualitativo en lugar de cuantitativo y escritos diferentes a un artículo científico como actas de congresos o ensayos breves (Rodríguez, Rodríguez, Rodríguez G, \& Bohórquez, 2021).

\subsection{Selección de los estudios}

Se realizó una lectura del título y del resumen de los trabajos que habian sido seleccionados al cumplir los criterios de búsqueda que se han mencionado. Luego, se realizó una lectura comprensiva de los artículos y se examinaron recogiendo información del título, autores, año de publicación, características de la muestra e instrumentos. (Rodríguez, Rodríguez, Rodríguez G, \& Bohórquez, 2021).

La metodología utilizada es cuantitativa, porque parte de una recopilación numérica, donde se ha realizado un análisis estadístico para establecer pautas de comportamiento y de aprobación de teorías donde se aplican métodos estadísticos para extraer una serie de conclusiones (Cazallo, Bascón, Mudarra, \& Salazar, 2020) citan a (Hernández, Fernández \& Baptista, 2014). 


\subsection{Instrumentos}

Para recolectar los datos demográficos se utilizó una ficha de caracterización que incluyó las variables de género, edad, formación académica y correo, mientras que para la evaluación de la inteligencia emocional se utilizó el modelo ECI (Emotional Competence Inventory) (Astudillo, 2019), propuesto por Goleman, debido al gran impacto causado en el contexto social por su propuesta al entorno de jóvenes, como apoyo a aquellos desertores del sistema educativo regular, y que lo ratifica la autora López (2016) en su investigación donde el instrumento de medición ECI abarca áreas de educación, salud administración entre otras, ya que así se les brinda una oportunidad de desarrollar competencias emocionales, para que logren aceptarse a sí mismos, como también el hecho de desarrollar la empatía y de esta manera sea factible manejar situaciones conflictivas (Fernández \& Inga, 2020).

Así mismo se eligió el modelo mixto del autor Goleman, ya que en el entorno universitario es muy útil la elaboración de un perfil al basarse en los rasgos de personalidad, porque permite visualizar al individuo evaluado al momento de evidenciar factores relacionados al aspecto emocional de la persona (Macavilca, 2020).

La escala consta de 29 preguntas relacionadas a Autoconciencia, Autocontrol, Empatía y Habilidades Sociales agrupados en 4 variables, en la cual los evaluados responden a cada ítem según las opciones de respuestas: "Siempre", “a veces" y "nunca” (ver Tabla 1. Sistema de Variables). (Ugarriza, 2003) citado por (García, Hurtado, Quintero, Rivera, \& Ureña, 2018).

Los participantes en esta investigación en contexto se tomaron de una muestra formada por 160, desglosados en dos grupos: 47 del grupo 
masculino y 113 del grupo femenino, pertenecientes a la facultad de medicina de la carrera de enfermería de la Universidad UNEMI.

Tabla 1 Variables

\begin{tabular}{|c|c|c|c|c|}
\hline Instrumento & Variable & Medición & Tipo & Dimensión \\
\hline \multirow{8}{*}{$\begin{array}{c}\text { Datos } \\
\text { demográficos }\end{array}$} & \multirow[b]{2}{*}{ Género } & \multirow[b]{2}{*}{ Cualitativa } & \multirow[b]{2}{*}{ Nominal } & Masculino \\
\hline & & & & Femenino \\
\hline & \multirow{4}{*}{ Edad } & \multirow{4}{*}{ Cuantitativa } & \multirow{4}{*}{ Intervalo } & 18 a 29 años \\
\hline & & & & 30 a 40 años \\
\hline & & & & 40 a 50 aก̃os \\
\hline & & & & 51 o más \\
\hline & \multirow{2}{*}{$\begin{array}{l}\text { Conoce sobre } \\
\text { Inteligencia } \\
\text { emocional }\end{array}$} & \multirow[b]{2}{*}{ Cualitativa } & \multirow[b]{2}{*}{ Nominal } & $\mathrm{Si}$ \\
\hline & & & & No \\
\hline \multirow{2}{*}{$\begin{array}{l}\text { Emotional } \\
\text { Competence }\end{array}$} & Autoconciencia & Cualitativa & \multirow{4}{*}{ Nominal } & \multirow{4}{*}{$\begin{array}{l}\text { Siempre } \\
\text { A veces } \\
\text { Nunca }\end{array}$} \\
\hline & Autocontrol & Cualitativa & & \\
\hline \multirow{2}{*}{$\begin{array}{c}\text { Inventory, } \\
\text { Goleman, } 2008\end{array}$} & Empatía & Cualitativa & & \\
\hline & $\begin{array}{l}\text { Habilidades } \\
\text { Sociales }\end{array}$ & Cualitativa & & \\
\hline
\end{tabular}

Fuente: Autores

\subsection{Consideraciones Éticas}

En la presente investigación se tendrá en cuenta el Código Ético de la APA, a pesar de que su vigencia es en EUA, también se menciona la IUPsyS, adoptada por la Asamblea de la Unión Internacional de Ciencia Psicológica en Berlín, el 22 de julio de 2008, cuyo fin considera el bienestar de la sociedad atribuyendo principios éticos para mejorar la calidad de vida de los seres humanos.

El presente trabajo de investigación tendrá como objetivo medir la Inteligencia emocional en estudiantes hombres y mujeres de la Facultad de Enfermeria de la UNEMI. 


\subsection{Riesgos y Beneficios}

- Este estudio no implicará ningún riesgo físico o psicológico para los participantes.

- Las respuestas dadas en la prueba no ocasionarán ningún riesgo, ni tendrán consecuencias en la situación académica o reputación de los participantes.

- La participación en la investigación no generará ninguna clase de gastos económicos.

- Los participantes no recibirán ningún beneficio académico o económico por contribuir en este estudio.

- Este estudio no tiene ningún interés económico por parte de la Facultad de Enfermería, ni de los estudiantes, ni profesores de la institución.

\subsection{Derechos}

La participación en la presente investigación es absolutamente voluntaria. Los participantes podrán elegir si desean intervenir o no en el estudio, en caso de aceptar los estudiantes podrán retirarse en cualquier momento durante la aplicación de la prueba sin recibir ninguna amonestación.

\subsection{Uso y Confidencialidad de los Datos}

Toda la información concerniente a la prueba será confidencial y no será divulgada ni entregada a ninguna otra institución o individuo sin el consentimiento expreso del participante. Tampoco se contempla la entrega de resultados individuales del estudio. 


\section{Resultados}

\subsection{Caracteristicas Sociodemográficas}

Se analizaron 160 encuestas que corresponden a 113 estudiantes femeninos y a 47 estudiantes masculinos como se aprecia en la gráfica No. 4.

Gráfica No. 4 Encuestados por género

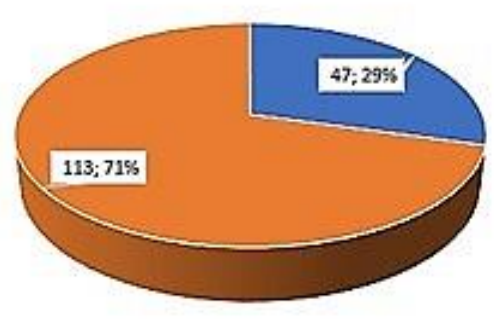

- Masculino = Femerino

Fuente. N. 170 Aplicación del Test Inteligencia Emocional

Dentro de las variables demográficas se puede identificar que de las 160 personas el $29 \%$ pertenecen al género masculino y el $71 \%$ son del sector femenino.

Tabla 2 Edad

\begin{tabular}{|l|r|r|}
\hline \multicolumn{1}{|c|}{ Edad } & \multicolumn{1}{|c|}{ Frecuencia } & \multicolumn{1}{|c|}{ Porentaje } \\
\hline De 18-29 & 155 & 96.9 \\
\hline 3040 años & 4 & 2.5 \\
\hline $41-50$ años & 1 & 0.6 \\
\hline 510 maja & 0 & 100 \\
\hline Total & 160 & \\
\hline
\end{tabular}

En la Tabla 2 se puede identificar que el 96.9\% (155 personas) tienen una edad comprendida entre los 18 a 29 años, el 2.5\% (4 personas) tienen 
una edad comprendida entre los 30 y 40 años, y el 0.6\% (1 persona) tiene una edad comprendida entre los 41 a 50 años. Esto indica que los adultos jóvenes e intermedios buscan realizar estudios en el área de enfermería.

Tabla 3. Inteligencia Emocional Componente IntrapersonalAutoconciencia

\begin{tabular}{|c|c|c|c|c|c|c|c|}
\hline \multirow[t]{2}{*}{ Indicador } & \multirow[t]{2}{*}{ Item| } & \multicolumn{2}{|c|}{ Siempre } & \multicolumn{2}{|c|}{ A reces } & \multicolumn{2}{|c|}{ Nunca } \\
\hline & & $\mathrm{N}$ & $\%$ & $\mathrm{~N}$ & $\%$ & $N$ & $\%$ \\
\hline \multirow[t]{2}{*}{$\begin{array}{l}\text { Conocimiento } \\
\text { de sí mismo }\end{array}$} & $\begin{array}{l}\text { Soy capaz de percibir la relación } \\
\text { que hay entre mis pensamientos } \\
\text { y sentimientos }\end{array}$ & 70 & 44 & \$S & 53.5 & 4 & 2.5 \\
\hline & $\begin{array}{l}\text { Anticipo los efectos que pueden } \\
\text { tener mis emociones en los } \\
\text { demás }\end{array}$ & 54 & 34 & 95 & 59.7 & 10 & 6.3 \\
\hline \multirow[t]{2}{*}{ Autoeraluación } & $\begin{array}{l}\text { Soy capaz de reconocer mis } \\
\text { fortalezas para emplearlas en el } \\
\text { logro de mis metas }\end{array}$ & 106 & 66.7 & 46 & 28.9 & 7 & 4.4 \\
\hline & $\begin{array}{l}\text { Siento satisfacción cada vez que } \\
\text { realizo cualquier actividad } \\
\text { dentro o fuera del aula }\end{array}$ & 121 & 76.1 & 34 & 21.4 & 4 & 2.5 \\
\hline \multirow[t]{2}{*}{$\begin{array}{l}\text { Confianza en sí } \\
\text { mismo }\end{array}$} & $\begin{array}{l}\text { Me arriesgo a tomar decisiones } \\
\text { firmes a pesar de las presiones }\end{array}$ & 76 & 47.8 & 76 & 47.8 & 7 & 4,4 \\
\hline & $\begin{array}{l}\text { Confio en mis habilidades para } \\
\text { resolver un conflicto con mis } \\
\text { compañeros }\end{array}$ & $\$ 9$ & 56 & 67 & 42.1 & 3 & 1.9 \\
\hline
\end{tabular}

Al analizar las respuestas emitidas en la tabla 3 en el indicador Conocimiento de sí mismo, se identificó que un 53.5\% de los estudiantes señalan que algunas veces son capaces de percibir la relación entre sus pensamientos y sentimientos, por su parte $59.7 \%$, manifiestan que a veces pueden anticipar los efectos que pueden tener sus emociones en los demás., lo cual conduce a señalar que muchos de ellos tienen algunas dificultades para saber si sus sentimientos y pensamientos están o no 
influyendo en sus relaciones intrapersonales y como referente para la toma de decisiones acertadas dentro del contexto socio-escolar.

En el caso del indicador Autoevaluación se expresa que el 66.7\% de los estudiantes siempre son capaces de reconocer sus fortalezas para emplearlas en el logro de las metas y a la vez, siempre el 76.1\% se siente satisfecho cada vez que realiza cualquier actividad dentro o fuera del aula., lo cual permite inferir que la mayoría de ellos, tienen la suficiente capacidad para reconocer que sí poseen las fortalezas para afrontar cualquier reto en su vida personal o estudiantil.

Para el indicador Confianza en sí mismo se evidencia un empate de los estudiantes encuestados entre la opción de respuesta: siempre y algunas veces se arriesga a tomar decisiones firmes a pesar de las presiones; de igual manera, un 56\% registró que siempre confia en sus habilidades para resolver un conflicto con sus compañeros, lo que denota que tienen una percepción de confianza en sí mismos más de la mitad de los alumnos. 
Tabla 4. Inteligencia Emocional Componente IntrapersonalAutocontrol

\begin{tabular}{|c|c|c|c|c|c|c|c|}
\hline \multirow[t]{2}{*}{ Indicador } & \multirow[t]{2}{*}{ Item } & \multicolumn{2}{|c|}{ Siempre } & \multicolumn{2}{|c|}{ A veces } & \multicolumn{2}{|c|}{ Nmea } \\
\hline & & $\mathbf{N}$ & $\%$ & $\mathbf{N}$ & $\%$ & N & $\%$ \\
\hline \multirow{2}{*}{$\begin{array}{l}\text { Control de } \\
\text { comportami } \\
\text { ento }\end{array}$} & $\begin{array}{l}\text { Mantengo la serenidad ante situaciones } \\
\text { dificiles con mis compañeros en el aula }\end{array}$ & 100 & 62.5 & 56 & 35 & 4 & 25 \\
\hline & $\begin{array}{l}\text { Cuando cometo un error estoy dippuesto } \\
\text { a reconocerlo }\end{array}$ & 131 & 81.9 & 29 & 18.1 & 0 & 0 \\
\hline \multirow[t]{2}{*}{$\begin{array}{c}\text { Autodomini } \\
\circ\end{array}$} & $\begin{array}{l}\text { Mamejo adecuadamente mis impulxos } \\
\text { ante un dexacuardo con mix compañeros }\end{array}$ & 102 & 63.7 & 55 & 34.4 & 3 & 19 \\
\hline & $\begin{array}{l}\text { Reflexiono sobre cómo expresar en un } \\
\text { momento determinado mis emociones } \\
\text { de: rabia, irs, angurtiz, inceridumbre, } \\
\text { etc. }\end{array}$ & 83 & 51.9 & 71 & 44.4 & 6 & 3.7 \\
\hline \multirow[t]{3}{*}{$\begin{array}{l}\text { Manejo de } \\
\text { ansiedad }\end{array}$} & $\begin{array}{l}\text { Se me dificulta coordinar ideas claras } \\
\text { cuando me siento presionado por las } \\
\text { actividadex encolares }\end{array}$ & 41 & 25.6 & 102 & 63.7 & 17 & 10.6 \\
\hline & $\begin{array}{l}\text { Me giento angurtiado cuando tengo que } \\
\text { enfrentar exposiciones }\end{array}$ & 56 & 35 & 79 & 49.4 & 25 & 15.6 \\
\hline & $\begin{array}{l}\text { Se me dificulta mantener la calma ante la } \\
\text { diversidad de eusluacionex o } \\
\text { axignariones que debo pregentar }\end{array}$ & 36 & 22.5 & 93 & 58.1 & 31 & 19.4 \\
\hline
\end{tabular}

En este análisis, en la tabla 4, se percibe en cuanto al control de Comportamientos que un $62.5 \%$ mantiene la serenidad en situaciones dificiles y que un $81.9 \%$ está dispuesto a reconocer si cometió un error. Se puede evidenciar que la gran mayoría de estudiantes sí disponen de la capacidad de controlar su comportamiento en el aula de clase, de reconocer cuando están equivocados y mantener la serenidad cuando enfrentan momentos dificiles. 
En relación con el indicador Autodominio, el 34.4\% manifiestan que algunas veces manejan adecuadamente sus impulsos ante un desacuerdo con sus compañerosy el 63.7 manifiestan que siempre. Así mismo el $49.4 \%$, algunas veces reflexionan sobre cómo expresar en un momento determinado sus emociones de rabia, ira, angustia, incertidumbre, entre otras. $\mathrm{Y}$ un promedio de $51.9 \%$ aseveran que siempre tienen dominio de las emociones, lo que permite inferir que la minoría presenta dificultades para controlar los impulsos y en un momento determinado puedan perder el control y estallar emocionalmente.

Por su parte al analizar el indicador Ansiedad, el $63.7 \%$ expresan que algunas veces se les dificulta coordinar ideas claras cuando se sienten presionados por las actividades escolares, y que sólo el 25.6\% siempre se sienten presionados, el 49.4\% a veces sienten angustia cuando tienen que exponer y sólo el 35\% siempre siente angustia al exponer, y solo un $15.6 \%$ nunca siente angustia al exponer, y solo el $19.4 \%$ de los encuestados admite que tiene la capacidad de manejar la ansiedad durante el desarrollo de cualquier actividad académica (evaluaciones de trabajos académicos, exposiciones, etc.); por lo que se infiere que muchos de los adolescentes demuestran desajustes emocionales cuando deben presentar una tarea o evidencia de aprendizaje que se le ha solicitado. 
Tabla 5 Inteligencia Emocional Componente Intrapersonal- Empatía

\begin{tabular}{|c|c|c|c|c|c|c|c|}
\hline \multirow[t]{2}{*}{ Indicador } & \multirow[t]{2}{*}{ Item } & \multicolumn{2}{|c|}{ Siempre } & \multicolumn{2}{|c|}{ A veces } & \multicolumn{2}{|c|}{ Ymea } \\
\hline & & $\mathbf{N}$ & $\%$ & $\mathbf{N}$ & $\%$ & $\mathbf{N}$ & $\%$ \\
\hline \multirow[t]{2}{*}{$\begin{array}{l}\text { Interéa por } \\
\text { los demás }\end{array}$} & $\begin{array}{l}\text { Comprendo las necesidades de mis } \\
\text { compañeros de aula }\end{array}$ & $10 \mathrm{~s}$ & 67.5 & 52 & 32.5 & 0 & 0 \\
\hline & $\begin{array}{l}\text { Me intereso por laz necenidades de mix } \\
\text { compañeros }\end{array}$ & 95 & 59.4 & 62 & 38.7 & 3 & 19 \\
\hline \multirow{2}{*}{$\begin{array}{l}\text { Orientació } \\
\text { n hacia el } \\
\text { servicio }\end{array}$} & $\begin{array}{l}\text { Offerco apoyo a mix compañeros de } \\
\text { clase con agrado }\end{array}$ & 119 & 74.4 & 40 & 25 & 1 & 0.6 \\
\hline & $\begin{array}{l}\text { Ofrezco mi apoyo ineondicional para } \\
\text { ayudar a mis compañeros en la } \\
\text { resolusión de problemas }\end{array}$ & 100 & 62.9 & 57 & 35.8 & 2 & 13 \\
\hline \multirow[t]{3}{*}{$\begin{array}{l}\text { Saber } \\
\text { escuchar }\end{array}$} & $\begin{array}{l}\text { Eacucho con atención a mix compañeros } \\
\text { de clase para entender sux gentimientos }\end{array}$ & 105 & 65.6 & 53 & 33.1 & 2 & 12 \\
\hline & $\begin{array}{l}\text { Me guata excurhar lax opiniones de mis } \\
\text { compañeros de clage en relación a un } \\
\text { trabajo de aula para gacar la conclusión } \\
\text { final| }\end{array}$ & 131 & 81.9 & 28 & 17.5 & 1 & 0.6 \\
\hline & $\begin{array}{l}\text { Escucho con farilidad los desacuerdos } \\
\text { que se presentan en el grupo }\end{array}$ & 117 & 73.1 & 42 & 26.2 & 1 & 0.6 \\
\hline
\end{tabular}

En el análisis del indicador Interés por los demás el $67.5 \%$ de los estudiantes señalan que siempre comprenden las necesidades de sus compañeros de aula, así mismo el $59.4 \%$ señalan que siempre se interesan por las necesidades de éstos, permitiéndoles comprender sus necesidades. Por todo ello se infiere que más de la mitad de los estudiantes se involucran en brindar apoyo y comprender por lo que están atravesando sus compañeros de grupo. 
En este contexto en relación con el indicador Orientación hacia el servicio, el $74.4 \%$ de la muestra, manifiesta que siempre tienen un acercamiento afectivo con sus compañeros y el 62.9\% siempre ofrecen su incondicional apoyo en situaciones académicas; todo ello evidencia que existe un marcado interés personal, donde la mayoría se centran en resolver sus problemas y se involucran en brindar apoyo y comprender por lo que están atravesando sus compañeros de grupo, demostrando apoyo, sentido de compromiso y compañerismo; evidenciando empatía que prevalece en la mayoría de los estudiantes objeto de estudio.

Así mismo al analizar el indicador saber escuchar el $65.6 \%$ de los estudiantes objeto de estudio, manifiestan que siempre escuchan con atención a sus compañeros de clase para entender sus sentimientosy asî mismo el $81.9 \%$ refieren que siempre les gusta escuchar las opiniones de sus compañeros de clase en relación con un trabajo de aula para sacar la conclusión final, a la vez el $73.1 \%$ siempre escucha con facilidad los desacuerdos que se presentan en el grupo, lo que contribuye a mejorar las relaciones interpersonales, por cuanto la mayoria se muestra sensible hacia los puntos de vista de los otros para brindar ayuda en la comprensión de las necesidades y sentimientos de los demás. 
Tabla 6 Inteligencia Emocional Componente IntrapersonalHabilidades Sociales

\begin{tabular}{|c|c|c|c|c|c|c|c|}
\hline \multirow[t]{2}{*}{ Indicador } & \multirow[t]{2}{*}{ Item } & \multicolumn{2}{|c|}{ Siempre } & \multicolumn{2}{|c|}{ A reces } & \multicolumn{2}{|c|}{ Nmea } \\
\hline & & N & $\%$ & $\mathbf{N}$ & $\%$ & $\mathbf{N}$ & $\%$ \\
\hline \multirow[t]{3}{*}{$\begin{array}{l}\text { Prácticas } \\
\text { de } \\
\text { liderazgo }\end{array}$} & $\begin{array}{l}\text { Ante una situación conflictiva en el aula } \\
\text { propongo altenativas gencillas de } \\
\text { solnción }\end{array}$ & 57 & 35.6 & 86 & 53.8 & 17 & 10.6 \\
\hline & $\begin{array}{l}\text { Participolactivamente en las elecciones } \\
\text { extudiantiles }\end{array}$ & 42 & 26.3 & 83 & 51.9 & 35 & 21.9 \\
\hline & $\begin{array}{l}\text { Ejerzo liderargo ectudiantil para } \\
\text { cooperar en la solución de los } \\
\text { problemag de la imstitución }\end{array}$ & 38 & 23.8 & 84 & 52.5 & 38 & 23.8 \\
\hline \multirow[t]{2}{*}{$\begin{array}{l}\text { Resolución } \\
\text { de } \\
\text { conflictos }\end{array}$} & $\begin{array}{l}\text { Mantengo una actitud de confianza para } \\
\text { resolver situariones conflictivas en el } \\
\text { aula de clage }\end{array}$ & 80 & so & 62 & 38.7 & 11.3 & 11.3 \\
\hline & $\begin{array}{l}\text { En un problema con mis compañeros } \\
\text { formulo lineamientos para rexolverlos }\end{array}$ & 72 & 45 & 74 & 46.3 & 14 & 8.8 \\
\hline \multirow[t]{2}{*}{$\begin{array}{l}\text { Trabajo en } \\
\text { equipo }\end{array}$} & $\begin{array}{l}\text { Comparto actividades excolares } \\
\text { información y/o recursos con los } \\
\text { compañeros de clape }\end{array}$ & 78 & 48.8 & 76 & 47.5 & 6 & 3.7 \\
\hline & $\begin{array}{l}\text { Me gurta conformar equipos de trabajo } \\
\text { con mis amigos para realizar las } \\
\text { actividader egcolares }\end{array}$ & 107 & 66.9 & 45 & 28.1 & 8 & 5 \\
\hline \multirow[t]{2}{*}{$\begin{array}{l}\text { Comumicac } \\
\text { ión eficaz }\end{array}$} & $\begin{array}{l}\text { Exprego con facilidad los desacuerdos } \\
\text { que se presentan en mi Erupo de trabajo }\end{array}$ & 75 & 46.9 & 74 & 46.3 & 11 & 69 \\
\hline & $\begin{array}{l}\text { Comumico la confianza que tengo en } \\
\text { mis compañeros para alcanzar las metas }\end{array}$ & 97 & 60.6 & 58 & 36.3 & 5 & 3.1 \\
\hline
\end{tabular}

Como se puede observar en relación con el indicador Prácticas de liderazgo, solo el $53.8 \%$ responde algunas veces en participar ante situaciones de conflicto en el grupo de clase, donde proponen alternativas para su solución, y sólo el 51.9\% algunas veces participan activamente en elecciones estudiantiles, mientras que el $52.5 \%$ algunas veces ejercen liderazgo estudiantil para cooperar en la solución de los problemas de la institución o conflicto que altere el ambiente del aula. 
Para el indicador Resolución de conflictos el 50\% de los estudiantes manifiestan que siempre mantienen una actitud de confianza para resolver situaciones conflictivas en el aula de clase y el $46.3 \%$ algunas veces y ante un problema con sus compañeros formulan lineamientos para resolverlos. Al analizar esta categoría, en promedio menos de la mitad de las estudiantes, siempre contribuyen a la resolución de conflicto lo cual influye de cierta manera en el clima hostil del aula.

Por su parte en el indicador Trabajo en equipo el $48.8 \%$ de los estudiantes refiere que siempre comparten actividades escolares información y/o recursos con los compañeros de clase y el 47.5\%, manifiestan que lo hacen algunas veces. Así mismo el 66.9\% refiere que siempre les gusta conformar equipos de trabajo con sus amigos para realizar las actividades escolares y el $28.1 \%$ que solo algunas veces; lo cual demuestra que es favorable el desarrollo de las relaciones interpersonales, así como la formación de lazos afectivos entre ellos.

Al analizar el indicador Comunicación eficaz el $46.9 \%$ de los estudiantes siempre expresan con facilidad los desacuerdos que se presentan en su grupo de trabajo y el 46.3\%, refieren que a veces; y un $60.6 \%$ señala que siempre comunica la confianza que tiene en sus compañeros para alcanzar las metas trazadas por el grupo. En este ámbito se puede aseverar que sólo un promedio del 33.3\% siempre mantiene una comunicación eficaz en el aula de clase, por lo que se pone de manifiesta la alta comunicación entre los miembros del grupo clase.

\section{Discusión}

Vale mencionar que el instrumento utilizado para evaluar la inteligencia emocional que se ha descrito (ECI) versión anterior a ESCI (HayGroup, 2011), no evalúa todos los aspectos posibles de la inteligencia 
emocional, capta sólo una parte de lo que conceptualmente se entiende por ella (en especial, las 4 dimensiones o enfoques de Goleman), pero que, de acuerdo a muchos expertos, han sido aceptados como una herramienta para evaluar la IE (Trujillo \& Rivas, 2005; Villazhiñay, 2019).

Por otro lado, a pesar de que la sociedad ha dado mucha importancia a la inteligencia por encima de otras cualidades, esto no es condición suficiente que garantice el éxito profesional, académico o personal. Debido a que numerosos estudios han demostrado que existe una relación positiva entre inteligencia emocional y el rendimiento académico (Nieves, 2020), idea contrapuesta de un estudio realizado por la doctora Barchard (2003) citado por Astte \& Valdivia (2018), donde se evaluó la propuesta de Goleman que relacionaba el éxito académico y la IE y se demostró que la IE no era un predictor del éxito académico sino más bien la Habilidad Verbal (cognitiva) y la personalidad al 99\% de confianza.

Así también como lo mencionan los autores Astte \& Valdivia (2018) que citan a Goleman (1998), afirmando que una competencia emocional es una capacidad aprendida, por ende, las habilidades laborales y educativas pueden y deben aprenderse.

Goleman afirma también que la inteligencia emocional tiene un impacto en el rendimiento académico, como lo indica su libro Triple Focus (Goleman \& Senge, 2016) en donde el componente autocontrol le permite un buen desempeño escolary que la empatía le permite alcanzar el éxito académico según lo afirman los autores Astte \& Valdivia (2018), situación demostrada en la respuesta de los formularios llenada por los estudiantes de la presente investigación.

El modelo de Goleman incluye un conjunto de competencias emocionales dentro de cada construcción de inteligencia emocional, él opinó que las competencias emocionales no son talentos innatos, sino 
capacidades aprendidas en las que se debe trabajar y desarrollador para lograr un rendimiento sobresaliente (Fernández \& Inga, 2020), cuyo concepto adquiere mayor importancia como lo afirma Goleman, que la IE es el mejor indicador del éxito vital (Parra, Ramírez, Pernas, \& Ortiz, 2018).

\section{Conclusiones}

En el presente estudio se concluye que la mayoría de los estudiantes de la carrera de Licenciatura en Enfermería de la UNEMI, presentan un nivel de Inteligencia Emocional medio - alto, lo que denota una personalidad más plena y satisfactoria de la vida como estudiante, teniendo la posibilidad de convertirse en un profesional competente y de calidad.

Con base a la información obtenida de las encuestas se concluye que la inteligencia emocional se maneja de manera efectiva por el género femenino en comparación con la población masculina, por lo que los autores Astte \& Valdivia (2018) que citan a Goleman (1998), afirman que una competencia emocional es una capacidad aprendida, por ende, las habilidades laborales y educativas pueden y deben aprenderse.

No obstante, las ventajas de esta forma de medición son indudables, sobre todo en combinación con otros instrumentos. A través de los cuales, se obtienen indicadores de ejecución en habilidades emocionales concretas que pueden ser educadas y entrenadas posteriormente, lo que permite, además evitar sesgos perceptivos que son más difíciles de distorsionar, debido a su formato, cuando el alumno trata de ofrecer una imagen más positiva de sí mismo.

Por lo anteriormente expuesto queda el reto de construir nuevos instrumentos que evalúen otros aspectos de la inteligencia emocional, 
puesto que, en relación a las medidas existentes como los aspectos interpersonales, incluso la IE es importante en personas que padecen de enfermedades crónicas (Parra, Ramírez, Pernas, \& Ortiz, 2018).

En ese sentido los autores (Alegría \& Sánchez, 2020) afirman que las aportaciones de esta investigación buscan mostrar cómo la inteligencia emocional, es primordial para que los alumnos y los profesores se relacionen y solucionen alguna situación o problema al establecer un comportamiento con empatía, como lo refiere el instrumento ESCI que es un excelente predictor de la habilidad social y emocional cuyas evidencias han sido demostradas en estudios realizados a estudiantes universitarios (HayGroup, 2011).

Así mismo el grupo docente buscará alguna estrategia para que todos los alumnos sean beneficiados de acuerdo con el nivel de manejo de emociones que ellos poseen (Benavidez \& Flores, 2019) cita a (Campos, 2014, p.15), aportando a su desempeño académico. Por consiguiente, al tener un buen control y desarrollo de las emociones; potencia el crecimiento personal y desarrollo de habilidades educativas.

Por último, la nueva generación de instrumentos deberá ser lo más realista posible y captar la idiosincrasia de cada cultura ya que, a pesar del esfuerzo de adaptación, los actuales han sido generados en el ámbito suramericano.

\section{Limitaciones Y Lineas Futuras De Investigación}

Entre las limitaciones del presente estudio, se puede mencionar, en el plano metodológico, el haber utilizado una muestra incidental en la cual la población está representada únicamente por estudiantes universitarios, siendo mayor la cantidad de mujeres en comparación a los hombres. Por este motivo, la generalización de los resultados es 
limitada. Futuros estudios buscarán ampliar las características socioeducativas de la muestra. 


\section{Referencias}

Alegria, M., \& Sánchez, D. (2020). Importancia de la inteligencia emocional y su impacto en la vida de los estudiantes de la educación superior. ISSN: 2007-7629. Obtenido de https://repository.uaeh.edu.mx/revistas/index.php/te pexi/article/view/5671/7286

Alejos, R., \& Cambero, S. (2017). Inteligencia Emocional en pacientes oncológicos según el sexo. Obtenido de Universidad Ricardo Palma, Perú: https://re positorio.urp.edu.pe/bitstream/handle/URP/1155/TESIS_ALEJ OS CAMBERO. pdf? sequence $=18$ sis Allowe $\mathrm{d}=\mathrm{y}$

Araya P, S., \& Espinoza, L. (abril de 2020). Aportes desde las neurociencias para la comprensión de los procesos de aprendizaje en los contextos educativos. doi:

http://dx.doi.org/10.20511/ pyr2020.v8n1.312

Astudillo, A. (2019). Inteligencia Emocional y desempeño laboral en los operarios de Distribuidora Droguería Las Américas. S.A.C. Trujillo. Obtenido de https:/ / dspace.unitru.edu.pe/bitstream/handle/UNITRU/15094/Astudillo\%20Mucha\%20An dr\%c3\%a9\%20Gustavo.pdf? sequence=18xis Allowe $\mathrm{d}=\mathrm{y}$

Astte, R., \& Valdivia, C. (2018). Inteligencia emocional y rendimiento académico en estudiantes de primaria de la ie $n^{\circ} 2094$ Inca Pachacútec de Lima. Obtenido de https:/ / re positorio.utelesup.e du.pe / bitstream/UTELESUP/635/1/ASTTE\%20TAPIA\%20ROS SANA\%20MARIA-VALDIVIA\%20CARHUAS\%20CARINA.pdf

Benavidez, V., \& Flores, R. (2019). La importancia de las emociones para la neurodidáctica. Obtenido de Rev. Estud. de Psicología UCR, 14(1) 2019 (Enero-Junio): 25-53 /ISSN: 1659-2107: https://dialnet.unirioja.es/descarga/articulo/6794283.pdf

Bueno, A. (2019). LA INTELIGENCIA EMOCIONAL: EXPOSICIÓN TEÓRICA DE LOS MODELOS FUNDANTES. Obtenido de p. 57-62: http://revistas.ut.edu.co/index.php/SyS/article/view/1816

Cáceres, M., García, R., \& G. (mayo de 2020). El manejo de la inteligencia emocional en los estudiantes de Secundaria. Un estudio exploratorio en una Telesecundaria en México. Revista Conrado, 16(74), 312-324. Obtenido de https://conrado.ucf.edu.cu/index.ph p/conrado/article/view/1367/1357

Cárdenas, D., \& Rodríguez, L. (2020). Inte ligencia emocional en estudiantes de Psicología en la Universidad Cooperativa de Colombia, Villavicencio. Obtenido de https://repository.ucc.edu.co/bitstream/20.500.12494/17545/4/2020_inteligencia_emocion al_estudiantes\%20\%282\%29.pdf

Castillo, R., García, V., Pena, M., \& Galán, A. B. (2017). Resultados preliminares del método RULER en la inteligencia emocional y el compromiso laboral de profe sores Españoles. doi: http://dx.doi.org/10.14204/ejrep.43.17068

Cazallo, A., Bascón, M., Mudarra, A., \& Salazar, E. (junio de 2020). La inteligencia emocional en los estudiantes universitarios. Obtenido de Revista spacios. ISSN: 0798-1015 . Vol. 41 (23) 2020 • Art. 28: https://www.revistaespacios.com/a20v41n23/a20v41n23p28.pdf

Estrada, E., Moysén, A., Balcazar, P., \& Garay, J. (Febrero de 2016). INTELIGENCIA EMOCIONAL EN ESTUDIANTES UNIVERSITARIOS MEXICANOS. Obtenido de XVII Congreso Virtual Internacional de Psiquiatría: http://ri.uaemex.mx/bitstream/handle/20.500.11799/65573/Interpsiquis\%202016\%20Inte ligencia\%20emocional $\% 20$ en $\% 20 \mathrm{j} \% \mathrm{C} 3 \%$ B3venes.pdf?sequence $=1$

Fernández, L., \& Inga, A. (2020). Análisis psicométrico del inventario de coeficiente emocional BarOn I- CE, en una prueba piloto para adolescentes de 17 a 18 años de edad de la Institución Fiscal Sucre y la Unidad Educativa Municipal del Milenio Bicentenario de la ciudad de Quito. 
Obtenido de http://www.ds pace.uce.edu.ec/bitstream/25000/21753/1/T-UCE-0010-FIL913.pdf

García A, C. (agosto de 2020). La inteligencia emocional en el de sarrollo de la trayectoria académica del universitario. Obtenido de ISSN 0257-4314:

http://scielo.sld.cu/scielo.php?script=sci_arttext\&pid=S0257-43142020000200015

García, M., Hurtado, P., Quintero, D., Rivera, D., \& Ureña, Y. (Junio de 2018). La ge stión de las emociones, una necesidad en el contexto educativo y en la formación profe sional. Obtenido de Vol. 39 ( $\mathrm{N}^{\circ}$ 49) Año 2018. Pág. 8. ISSN 0798 1015:

https://www.revistaespacios.com/a18v39n49/18394908.html\#tres

Godoy, I., \& Sánchez, M. (2020). Estudio sobre la Inteligencia Emocional en educación primaria. doi:1012795/revistafuentes.2021.12108

Goleman, D., \& Senge, P. (2016). Triple Focus. Editorial BDeBolsillo. Origen Argentina. ISBN 13 9788490703168.

Gutiérrez S, J., Zamora L, B., Fouilloux, C., \& Petra, I. (Mayo de 2021). Funciones mentales: neurobiologia. Obtenido de Departamento de Psiquiatría y Salud Mental, Facultad de Medicina, UNAM: http:/ / psiquiatria.facmed.unam.mx/wp-content/uploads/2021/05/2021Funciones-mentales-Neurobiologii\%CC\%80a.pdf

HayGroup. (2011). Emotional and social competency inventory (ESCI) A user guide for accredited practitioners. Obtenido de https://www.eiconsortium.org/pdf/ESCI_user_guide.pdfhttps://www.eiconsortium.org/pdf/ ESCI_user_guide.pdf

Leggiero, M.-P. (s.f). Impacto de las emocione s en el aprendizaje aúlico. Obtenido de https://www.te seopress.com/neurociencias/chapter/92/

López, J. (2016). Inteligencia Emocional en Docentes de la Universidad Militar Nueva Granda. Obtenido de https:/ / repository.unimilitar.edu.co/bitstream/handle/10654/14793/LOPEZ\%20PACHECO \%20JENNY\%20CAROLINA\%202016. pdf?sequence $=18$ is sAllowed=y

Macavilca, R. (2020). Vigencia de los modelos de inteligencia emocional para nuestros días. Obtenido de

https:/ / re positorio.upeu.edu.pe / bitstream/handle/20.500.12840/3768/Ruth_Trabajo_Bachi 11er_2020.pdf? sequence $=18$ is Allowe $\mathrm{d}=\mathrm{y}$

Mayer, J., \& Salovey, P. (1997). "What is emotional intelligence?" En P. Salovey y D. Sluyter. (Eds). Emotional Development and Emotional Intelligence: Implications for Educators pp. 3-31). New York: Basic Books

Montenegro, O. (octubre de 2020). La intelige ncia emocional y su efecto protector ante la ansiedad, de presión y el estrés académico en estudiantes universitarios.Rev. Tzhoecoen. doi: https:// doi.org/10.26495/tzh.v12i4.1395

Nieves, A. (2020). Inteligencia emocional percibida en estudiantes de educación superior: análisis de las diferencias en las distintas dimensiones. doi:10.15517/ap.v34i 128.34469

Parra, A., Ramírez, R., Pernas, I., \& Ortiz, A. (2018). La Inteligencia emocional en pacientes miembros de la Fundación Casa de la Diabetes. Obtenido de Revista Cubana Educación Superior. 2018. 2. 14-30: http://scielo.sld.cu/pdf/rces/v37n2/rces02218.pdf

Perestelo-Pérez, L. (2013). Standards on how to develop and report systematic reviews in Psychology and Health. International Journal of Clinical and Health Psychology, 13(1), 49-57.

Rodríguez, J., Rodríguez, A., Rodríguez G, J., \& Bohórquez, C. (2021). Inteligencia emocional y rendimiento académico en universitarios: una revisión sistemática. ISSN: 0798-1015. 
Obtenido de Vol. 42 (07) 2021 • Art. 10 . DOI: 10.48082/espacios-a21v42n07p10: https://www.revistaespacios.com/a21v42n07/a21v42n07p10.pdf

Saavedra, J., Díaz C, W., Zúñiga, L., Navia, C., \& Zamora, T. (2015). Correlación funcional de1 sistema límbico con la emoción, el aprendizaje y la memoria. Obtenido de Vol. 7 -No. 2: https://revistas.unal.edu.co/index.php/morfolia/article/view/52874/52541

Trujillo, M., \& Rivas, L. (2005). Orígenes, evolución y modelos de inteligencia emocional. Obte nido de ISSN 0121-5051: http:// www.scielo.org.co/scielo.php?script=sci_arttext\&pid=S0121 $\underline{50512005000100001}$

Viciana, V., Cano, L., Chacón, R., Padial, R., \& Martínez, A. (2017). Importancia de la motricidad para el desarrollo integral del niño en la etapa de educación infantil. Obtenido de Revista Digital de Educación Física. Año 8, Num. 47 - ISSN:1989-8304.:

http://emasf.webcindario.com

Villazhiñay, A. (2019). Percepciones de estudiantes de Psicología respecto a la teor ía y metodología de la inteligencia emocional. Obtenido de https:/ / dspace.ucuenca.edu.ec/bitstream/123456789/32928/3/Trabajo\%20de\%20titulaci\% C3\%B3n.pdf. 